OPEN ACCESS

Edited by:

Philippe Ovetchkine, University of Montreal, Canada

Reviewed by: llias Karaiskos,

Hygeia Hospital, Greece

Rinawati Rohsiswatmo, RSUPN Dr. Cipto

Mangunkusumo, Indonesia

*Correspondence:

Jianhong Chen

chenih-110@263.net

Specialty section: This article was submitted to Pediatric Infectious Diseases, a section of the journal Frontiers in Pediatrics

Received: 25 July 2020 Accepted: 29 June 2021 Published: 27 July 2021

Citation: Xing $H$, Cheng $C$, Zhang $Y$, Cai $Y$, Wang $X$, Deng $D, X U L, X \cup M$ and Chen J (2021) Successful Treatment With Intrathecal and Intravenous Polymyxin B-Based Combination

Against MDR Acinetobacter baumannii Meningitis in Pediatric Patient: A Case Report Front. Pediatr. 9:564991. doi: 10.3389/fped.2021.564991

\section{Successful Treatment With Intrathecal and Intravenous Polymyxin B-Based Combination Against MDR Acinetobacter baumannii Meningitis in Pediatric Patient: A Case Report}

\author{
Haiyan Xing ${ }^{1}$, Caiyi Cheng ${ }^{1}$, Yihua Zhang ${ }^{2}$, Yongqing Cai ${ }^{1}$, Xianfeng Wang ${ }^{1}$, \\ Dongmei Deng ${ }^{1}$, Lunshan $\mathrm{Xu}^{2}$, Minhui $\mathrm{Xu}^{2}$ and Jianhong Chen ${ }^{1 *}$
}

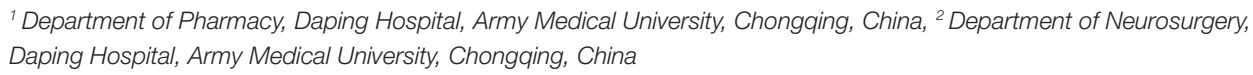

Background: Nosocomial meningitis with multidrug-resistant (MDR) or extensively drug-resistant (XDR) Acinetobacter baumannii is a life-threatening complication in neurosurgery. Treatment of these infections is challenging because of poor penetration of the available antibiotics into the cerebrospinal fluid (CSF). Intrathecal (ITH) or intraventricular (IVT) administration of antibiotics is increasingly used as the last treatment option against MDR/XDR Gram-negative bacteria meningitis not responding to intravenous (IV) regimens. However, pertinent data in pediatric patients is scarce.

Case Presentation: A 14-year-old male patient developed meningitis from an MDR strain of $A$. baumannii following endoscopic endonasal resection of craniopharyngioma. Despite a combination therapy involving IV tigecycline, we observed clinical and bacteriologic failure. The patient was then successfully treated with an ITH and IV polymyxin B-based combination. Quantification of tigecycline and polymyxin B in CSF was performed with two-dimensional high-performance liquid chromatography (2D-HPLC) and HDLC coupled with tandem mass spectrometry (HPLC-MS/MS), respectively. Adverse drug reactions (neurotoxicity and skin hyperpigmentation), probably induced by polymyxin B, were acceptable and reversible.

Conclusions: The case illustrates ITH and IV Polymyxin B-based combination is an optimal therapeutic option against MDR A. baumannii meningitis in this pediatric patient. In the future, real-time PK/PD data obtained from patients during ITH/IVT polymyxin B therapy should be required to optimize polymyxin use with maximal efficacy and minimal adverse effects.

Keywords: meningitis, multidrug resistance, Acinetobacter baumannii, Polymyxin B, tigecycline, intrathecal administration 


\section{INTRODUCTION}

Postoperative meningitis due to multidrug-resistant (MDR) or extensively drug-resistant (XDR) Gram-negative bacteria is a life-threatening complication in neurosurgery. Acinetobacter baumannii has emerged as one of the major organisms isolated from the cerebrospinal fluid (CSF) cultures (1). Mortality of nosocomial meningitis caused by $A$. baumannii was reported as high as $72.7 \%$ (2). MDR/XDR A. baumannii strains possess an impressive armamentarium of resistance mechanisms, resulting in resistance to all, or almost all, commercially available antibiotics (3). This fact has led to the resurrection of polymyxins (polymyxin B and colistin) as salvage therapy (4). Considering the potentially suboptimal pharmacokinetic $(\mathrm{PK}) /$ pharmacodynamic (PD) and the increasing prevalence of multidrug resistance, combination therapy is recommended for polymyxins even though there is a lack of solid clinical evidence (5-7). Furthermore, due to the poor central nervous system (CNS) penetration of antibiotics (8), intrathecal (ITH) or intraventricular (IVT) routes have been considered as the last resort for the treatment of ventriculitis/meningitis caused by MDR/XDR Gram-negative bacteria not responding to intravenous (IV) regimens (9). However, the clinical efficacy and tolerability profile of ITH/IVT polymyxins therapy, especially in pediatric patients, remains obscure.

This report presents a case of a 14-year-old male patient who developed meningitis from an MDR strain of A. baumannii following endoscopic endonasal resection of craniopharyngioma. The patient has been successfully treated with ITH and IV Polymyxin B-based combination after a failed combined therapy involving IV tigecycline. In addition, concentrations of tigecycline and polymyxin B in CSF were retrospectively determined by two-dimensional high-performance liquid chromatography (2D-HPLC) and HPLC coupled with tandem mass spectrometry (HPLC-MS/MS), respectively (Supplementary Material).

\section{CASE PRESENTATION}

The patient was admitted to our hospital on January 24, 2019, with blurred vision in both eyes for 1 week. Neurological examinations and laboratory evaluations revealed no abnormality. The patient was diagnosed with craniopharyngioma by neuroradiologic examinations (Figure 1A). Complete tumor removal was achieved via the endoscopic endonasal transsphenoidal approach on day 4. Perioperative IV antibiotic prophylaxis with ceftriaxone $(2 \mathrm{~g})$ was administered. The intraoperative course of the patient was uneventful. However, the postoperative MRI examination showed an incomplete skull base reconstruction on the following day (Figure 1B).

The immediate postoperative recovery of the patient was complicated by an episode of CSF rhinorrhoea. Ten days after surgery (day 14), the patient presented with remittent fever (peak at $39.6^{\circ} \mathrm{C}$, Figure $2 \mathrm{~A}$ ) and irritative cough. CSF rhinorrhoea was highly suspected. The patient thus underwent two transnasal endoscopic repairs (on day 14 and 31), in which fibrin glue, dural substitutes, autologous fat tissue graft, and temporary

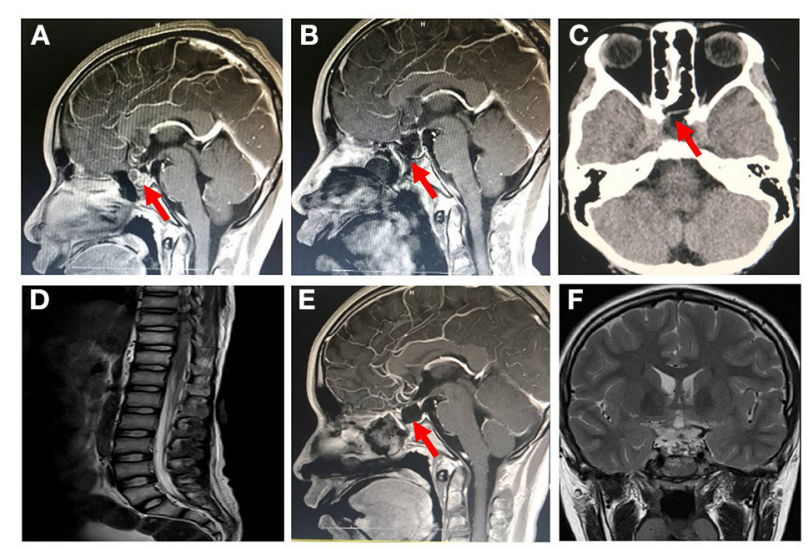

FIGURE 1 | Brain magnetic resonance imaging (MRI) and computed tomography (CT) image after patient admission. (A) Sagittal enhanced MRI of pituitary showed enlarged sella turcica with a mass of about $1.2 \mathrm{~cm} \times 1.6 \mathrm{~cm}$ $\times 1.4 \mathrm{~cm}$ in it, pituitary stalk extension to the right side and optic chiasm compression pituitary (January 25, 2019). (B) Sagittal enhanced MRI of pituitary showed that the craniopharyngioma was completely removed, and the skull base was incompletely covered by mucosal flap (January 29, 2019). (C) On day 47 of admission (March 12, 2019), nonenhanced skull CT scan revealed that the cerebrospinal fluid leakage was successfully repaired owing to the skull base reconstruction with mucosal flap. (D) Whole-spine MRI revealed no obvious adhesion in the spinal canal after 4 days of polymyxin B treatment (March 19, 2019). Follow-up pituitary MRI revealed (E) no recurrence of tumor, well healed mucosal flap in skull base, and (F) no obvious hydrocephalus (January 16, 2020).

lumbar drainage were used. The laboratory tests for CSF showed a slightly high level of white blood cell (WBC) and total cell counts (Table 1). In contrast, WBC count and neutrophilic granulocyte percentage (NEUT\%) in his peripheral blood were significantly higher (Figures 2B,C). Consequently, regardless of negative CSF culture, nosocomial meningitis was considered. IV linezolid (600 mg twice daily) and IV ceftriaxone ( $2 \mathrm{~g}$ twice daily) were initiated empirically (day 15). During follow-up, the patient's fever gradually subsided. However, a sudden onset of fever $\left(38.9^{\circ} \mathrm{C}\right)$ occurred on February 20th (day 27), with vomiting and neck rigidity. CSF analysis revealed a WBC count of $0.098 \times 10^{9} / \mathrm{L}$, a total protein of $2.41 \mathrm{~g} / \mathrm{L}$, and glucose of $2.32 \mathrm{mmol} / \mathrm{L}$ (Table 1). The simultaneous blood glucose level was $6.34 \mathrm{mmol} / \mathrm{L}$. CSF culture was positive for MDR A. baumannii (Table 2), which was sensitive to tigecycline, amikacin, and minocycline, and intermediate to cefoperazone/sulbactam based on the breakpoints from the Clinical and Laboratory Standards Institute (CLSI) (10). For tigecycline, the U.S. Food Drug Administration (FDA)-approved breakpoints were applied (11). Empiric treatment was quickly replaced by the therapeutic regimen of IV tigecycline (100 $\mathrm{mg} /$ day in two doses after a loading dose of $100 \mathrm{mg}$ ) combined with meropenem $(2 \mathrm{~g}$ as a 3 -h prolonged infusion every $8 \mathrm{~h}$ ) and amikacin $(7.5 \mathrm{mg} / \mathrm{kg}$ twice daily). CSF samples were collected from lumbar drainage before the initiation of the next dose of tigecycline and stored at $-80^{\circ} \mathrm{C}$ for uniform testing. Concentrations of tigecycline were determined by $2 \mathrm{D}$-HPLC with ultraviolet detection, and the 


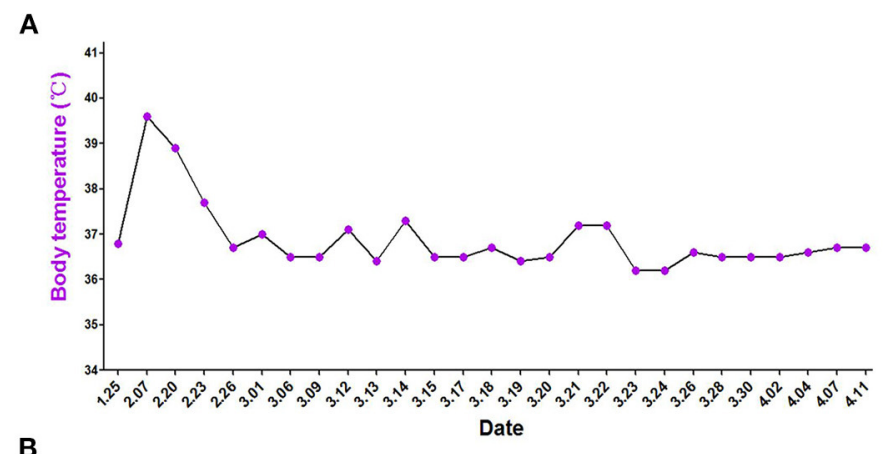

D

B
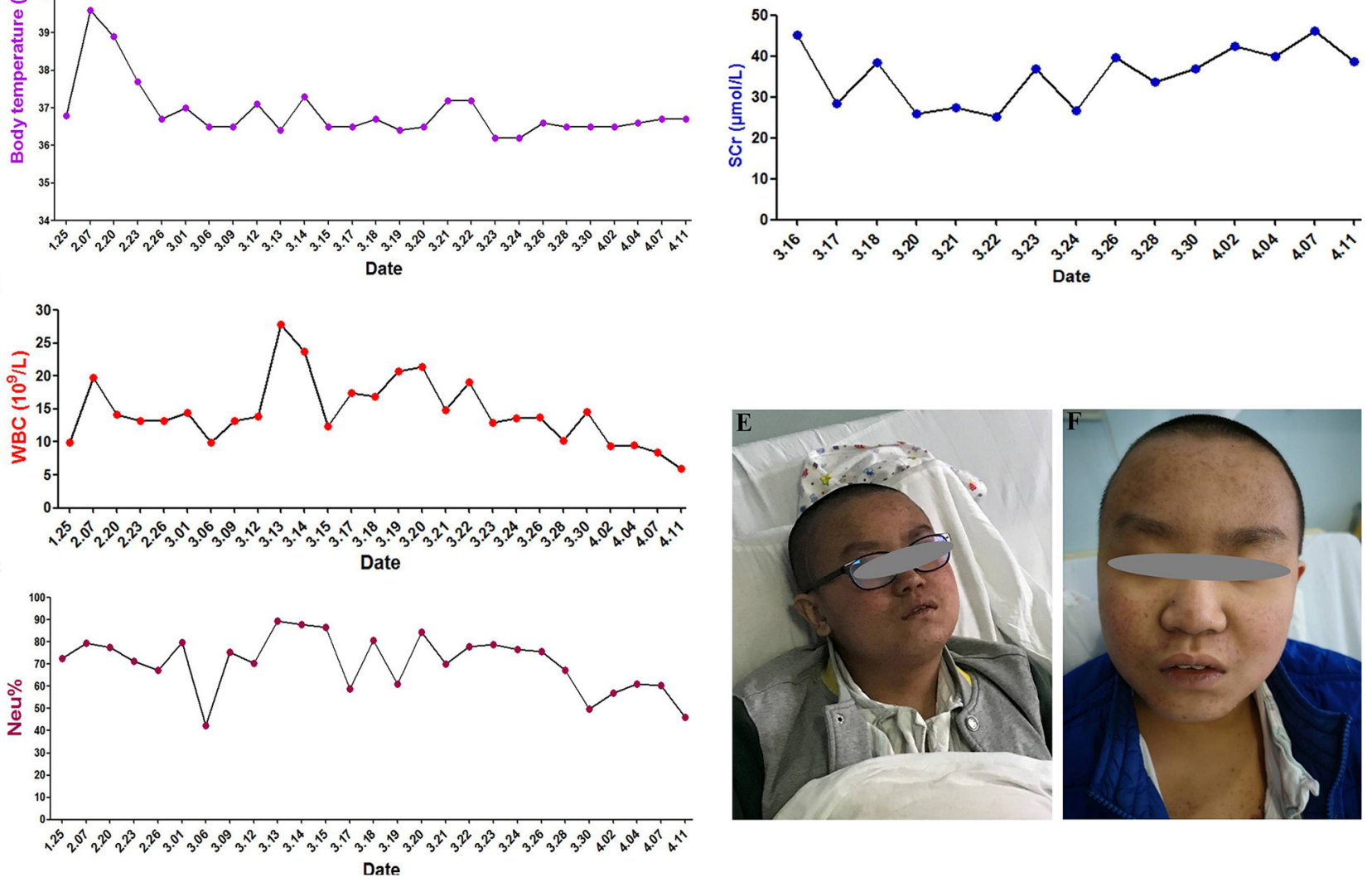

FIGURE 2 | Clinical course and skin hyperpigmentation of the patient. (A) Body temperature over the course of treatment. (B) White blood cell (WBC) count in the peripheral blood. (C) Neutrophil percentage (NEUT\%) in the peripheral blood. (D) Serum creatinine (SCr) levels. (E) Skin hyperpigmentation and dark red papule appeared in the upper part of trunk of patient on the fourth day of polymyxin B treatment (March 18, 2019). (F) Skin hyperpigmentation gradually disappeared (January 16, 2020).

signal was recorded at $340 \mathrm{~nm}$ (Figure 3A). Though the CSF cultures remained positive for A. baumannii, this therapy led to subsequent clinical and laboratory improvement. However, on day 46 after admission, the patient suffered from intolerable diarrhea without noticeable relief after symptomatic treatment. Considering the commonly reported side effects $(12,13)$, IV therapy with both tigecycline and amikacin was stopped (19 days from the start of IV tigecycline), while the IV meropenem was continued. Meanwhile, IV cefoperazone/sulbactam (3g every $6 \mathrm{~h}$ ) and minocycline (orally $200 \mathrm{mg} /$ day in two doses after a loading dose of $200 \mathrm{mg}$ ) were added. However, during treatment, gradually decreased CSF drainage was observed. Therefore, the lumbar drainage was removed, while a new sterile lumbar catheter was positioned on day 4 of this therapy. Simultaneously, CSF obtained from the new lumbar drainage was cloudy with a marked increase in protein $(3.65 \mathrm{~g} / \mathrm{L})$ and WBC count $(4.054 \times$ $10^{9} / \mathrm{L}$; Table 1 ), and the microbiological testing was consistently positive. Fortunately, a nonenhanced skull CT scan revealed successful repair of CSF leak (Figure 1C).

The antimicrobial therapy was changed to ITH polymyxin B (50,000 U once daily for 4 days, then $50,000 \mathrm{U}$ once every other day), IV polymyxin B (450,000 U twice daily), and cefoperazone/sulbactam (3 $\mathrm{g}$ every $6 \mathrm{~h}$ ) on March 15, 2019. Polymyxin B was dissolved in saline up to a total volume of $1 \mathrm{~mL}$ and slowly injected into the CSF (14). After each injection, the lumbar drainage was temporarily closed for $60 \mathrm{~min}$ to prevent untimely washout of the drug (1). CSF samples for laboratory examinations and concentration determinations were collected from the lumbar catheter before the next dose of polymyxin B. Direct quantification of polymyxin B in CSF was performed with HPLC-MS/MS equipped with electrospray (ESI) ionization interface (Figure 3B). CSF sterilization (a negative CSF culture) was achieved the next day, following remarkable improvement in CSF findings. Unfortunately, hyperpigmentation and dark red papule appeared in the upper part of the patient's trunk, especially on the face and neck on the fourth day of polymyxin B treatment, and gradually aggravated (Figure 2E). Over the next few days, the patient experienced mild memory loss, hypotonia, lumbosacral pain, and sore legs. However, whole-spine MRI revealed no apparent abnormality (Figure 1D), and renal function tests showed normal serum creatinine levels (Figure 2D). Because of the known neurotoxicity and skin hyperpigmentation induced 
TABLE 1 | Laboratory tests for cerebrospinal fuid over the course of treatment.

\begin{tabular}{|c|c|c|c|c|c|}
\hline Date & Cell count $\left(\times 10^{9} / \mathrm{L}\right)$ & WBC ( $\times 10^{9} /$ L) & Glucose (mmoL/L) & Total protein (g/L) & Chlorine (mmoL/L) \\
\hline 2019.02.07 (D14) & 0.040 & 0.019 & 3.30 & 0.27 & 119.0 \\
\hline 2019.02.20 (D27) & 2.075 & 0.098 & 2.32 & 2.41 & 112.4 \\
\hline 2019.02.26 (D33) & 0.643 & 0.187 & 1.95 & 0.79 & 120.7 \\
\hline 2019.03.01 (D36) & 0.018 & 0.015 & 1.88 & 0.65 & 122.0 \\
\hline 2019.03.09 (D44) & 0.009 & 0.004 & 2.90 & 0.16 & 121.9 \\
\hline 2019.03.11 (D46) & 0.008 & 0.002 & 4.40 & 0.14 & 120.9 \\
\hline 2019.03.14 (D49) & 4.142 & 4.054 & 0.33 & 3.65 & 118.5 \\
\hline 2019.03 .15 (D50) & 3.649 & 3.045 & 0.36 & 2.23 & 114.6 \\
\hline 2019.03.16 (D51) & 0.382 & 0.371 & 2.46 & 1.22 & 115.7 \\
\hline 2019.03.20 (D55) & 0.008 & 0.003 & 3.26 & 0.76 & 97.1 \\
\hline 2019.03.22 (D57) & 0.412 & 0.398 & 2.20 & 0.56 & 115.1 \\
\hline 2019.03.26 (D61) & 0.010 & 0.007 & 2.68 & 0.61 & 121.1 \\
\hline 2019.04.03 (D69) & 0.070 & 0.054 & 2.90 & 0.85 & 123.2 \\
\hline 2019.04.11 (D77) & 0.050 & 0.045 & 3.02 & 0.74 & 121.8 \\
\hline Normal reference ranges & $0-0.008$ & $0-0.008$ & $2.5-4.5$ & $0.15-0.45$ & $120-132$ \\
\hline
\end{tabular}

TABLE 2 | Antibiotics susceptibility tests for Acinetobacter baumannii in CSF.

\begin{tabular}{|c|c|c|c|}
\hline Antibiotics & Susceptibility & K-B (mm) & $\begin{array}{c}\text { MIC } \\
(\mu \mathrm{g} / \mathrm{mL})\end{array}$ \\
\hline Meropenem & Resistant & 8 & N/A \\
\hline Minocycline & Susceptible & 16 & $\mathrm{~N} / \mathrm{A}$ \\
\hline Cefoperazone/sulbactam & Intermediate & 18 & N/A \\
\hline Piperacillin & Resistant & 6 & $\mathrm{~N} / \mathrm{A}$ \\
\hline Amikacin & Susceptible & 22 & $\mathrm{~N} / \mathrm{A}$ \\
\hline Tigecycline & Susceptible & $\mathrm{N} / \mathrm{A}$ & 0.5 \\
\hline Ampicillin & Resistant & N/A & $\geq 32$ \\
\hline Cefazolin & Resistant & N/A & $\geq 64$ \\
\hline Ceftriaxone & Resistant & $\mathrm{N} / \mathrm{A}$ & $\geq 64$ \\
\hline Cefepime & Resistant & $\mathrm{N} / \mathrm{A}$ & $\geq 64$ \\
\hline Ceftazidime & Resistant & $\mathrm{N} / \mathrm{A}$ & $\geq 64$ \\
\hline Imipenem & Resistant & N/A & $\geq 16$ \\
\hline Piperacillin/tazobactam & Resistant & $\mathrm{N} / \mathrm{A}$ & $\geq 128$ \\
\hline Gentamicin & Intermediate & $\mathrm{N} / \mathrm{A}$ & 8 \\
\hline Tobramycin & Susceptible & $\mathrm{N} / \mathrm{A}$ & $\leq 1$ \\
\hline Ciprofloxacin & Resistant & $\mathrm{N} / \mathrm{A}$ & $\geq 4$ \\
\hline Levofloxacin & Resistant & $\mathrm{N} / \mathrm{A}$ & $\geq 8$ \\
\hline Trimethoprim-sulfamethoxazole & Resistant & N/A & 8 \\
\hline
\end{tabular}

by polymyxin B $(15,16)$, ITH polymyxin B was replaced with minocycline oral administration on March 24, 2019 (9 days from the onset of ITH polymyxin B), while IV polymyxin B and cefoperazone/sulbactam were continued. Ten days later, IV polymyxin B was discontinued. The signs of meningitis disappeared in a few days. Meanwhile, laboratory tests of CSF and radiological examination returned to normal. Thus, the patient was discharged on April 12, 2019.

At the 12-months follow-up, the patient was in good clinical condition without signs of intracranial infection. Neurologically, vision in both his eyes was normal. Skin hyperpigmentation (Figure 2F) and strength in his limbs were gradually recovered, and the patient could walk normally. Radiological signs were also normal (Figures 1E,F).

\section{DISCUSSION}

This paper reports on a 14-year-old male patient who developed MDR A. baumannii meningitis following neurosurgical surgery and subsequent CSF rhinorrhoea. The risk factors such as CSF leakage, lumbar drainage placement, and repeated transnasal endoscopic repair may be significantly associated with subsequent $A$. baumannii meningitis of this patient (17). Nosocomial meningitis due to MDR A. baumannii seriously threatens patient survival. However, its treatment is challenging because of limited available therapeutic options, especially in pediatric patients (3).

The frequent emergence of carbapenem resistance of $A$. baumannii has led to the revival in polymyxins and tigecycline as the last resort antimicrobials (18). Since polymyxins were not always available and the susceptibility test for polymyxin B and colistin were not routinely performed in our hospital, tigecycline was initially administered intravenously with meropenem and amikacin according to the in vitro susceptibility testing. Although 

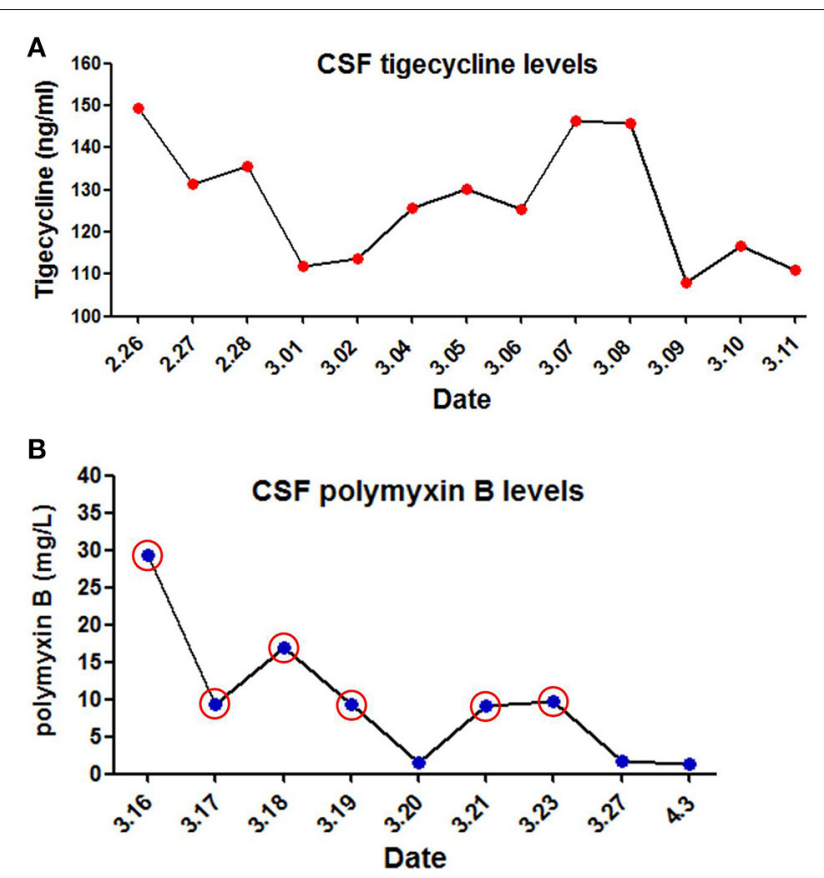

FIGURE 3 | Levels of tigecycline and polymyxin B in cerebrospinal fuid (CSF) of the patient. (A) CSF tigecycline concentrations were measured with Two-Dimensional High-Performance Liquid Chromatography (2D-HPLC) method. (B) CSF polymyxin B levels were determined using high performance liquid chromatography coupled with tandem mass spectrometry (LC-MS/MS). Red circle represents the day of ITH polymyxin B treatment.

tigecycline is not approved for use in children by FDA, the results of a few available data suggest that it may be a valauble consideration for life-threatening infections in pediatric patients, alone or in combination with other antibiotics, when other therapies are not suitable $(19,20)$. Moreover, there are some reported cases of postoperative meningitis that were successfully treated with tigecycline (the same dosage regimen as in the present case) as a last line therapy $(21,22)$. Hence, the combination of IV tigecycline with amikacin and prolonged infusion of meropenem may be a potential alternative for salvage treatment in this case.

Unfortunately, the tigecycline-based combination therapy failed to achieve microbiological eradication despite promising clinical and laboratory improvement. A previous study of tigecycline concluded that the administration of a dose of 1-2 $\mathrm{mg} / \mathrm{kg} \mathrm{q} 12 \mathrm{~h}$ in children aged 12 years or older, to a maximum dose of $50 \mathrm{mg}$, provided PK values similar to those reported to be effective in adults (23). However, our retrospective CSF determination showed that the steady-state concentration of tigecycline was $108.08-149.54 \mathrm{ng} / \mathrm{mL}$, a value far below the FDA breakpoint (24). It suggests that personalized dosing of IV administration of tigecycline is necessary for a patient with MDR A. baumannii meningitis to avoid suboptimal drug exposure in the CSF. Thus, further pharmacological studies are required to describe the $\mathrm{PK} / \mathrm{PD}$ profile of tigecycline in children of different ages and infection types to optimize the dosage regimen. Additionally, as described in recent studies, ITH/IVT administration of tigecycline may be another option for treating bacterial meningitis caused by $\operatorname{MDR}$ A. baumannii $(14,25)$. Regarding the safety profile, previous studies showed that the adverse events associated with tigecycline in children include nausea, vomiting, diarrhea, neutrophil engraftment delay, and acute pancreatitis (23). The patient in this case suffered from intolerable diarrhea leading to the discontinuation of tigecycline combination therapy. The result might also be attributed to other factors, such as a worse clinical condition and concomitant drugs (26). Therefore, additional data from controlled clinical studies are required to assess the safety of tigecycline, and particular caution should be given to off-label use of tigecycline, especially in pediatric patients.

Polymyxins have been revived as a last-line defense against difficult-to-treat MDR A. baumannii in this pediatric patient following the failure of IV tigecycline. Although limited polymyxin B information is available, especially in pediatric patients, recently available data indicates that polymyxin B has significant $\mathrm{PK} / \mathrm{PD}$ advantages in contrast to colistin, and its dosage regimens should not be adjusted according to the patient's renal function (27). As IV polymyxins demonstrate poor CNS penetration, ITH and IV routes have been employed to achieve high polymyxin concentrations in the CNS $(28,29)$. It is reported that the combination of IV and ITH/IVT polymyxins treatment has been used as an effective and safe therapeutic option against CNS infections caused by MDR Gram-negative bacteria, including in critically ill patients and children (30). Furthermore, polymyxin B monotherapy is not an ideal clinical option since dose escalation to achieve sufficiently high concentrations carries risks of rapid-onset nephrotoxicity (31). Thus ITH and IV polymyxin B-based combination was considered salvage therapy for this pediatric patient with lumbar drainage.

To the best of our knowledge the daily dose of polymyxins ranges between 5,000 IU (in infants) and 120,000 IU (divided into two doses) in children (32). The duration of therapy is quite variable ( $2-4$ weeks), and shorter treatments ( $<1$ week) may correlate with higher mortality (28). In this pediatric patient, the combination including a low dosage of ITH polymyxin B was administered. CSF sterilization was rapidly achieved without significant nephrotoxicity. However, the mild neurotoxicity and skin hyperpigmentation caused by polymyxin B was highly suspected, although it was difficult to isolate adverse reactions and clinical outcomes solely from this drug. As reported previously, the onset of polymyxin B-induced neurotoxicity and hyperpigmentation usually occurs shortly after IV infusion or within the first few days of polymyxin therapy and is generally reversible with drug discontinuation $(33,34)$. The major risk factors associated with neurotoxicity are the extended exposure (duration and concentration) to polymyxins; the presence of medical conditions such as myasthenia gravis, renal impairment, and hypoxia; concomitant use of other medications (e.g., sedatives, anesthetics, narcotics, and muscle relaxants); and gender (33). These findings are in accordance with the suspected adverse reactions observed in the present case. Moreover, recent case reports of polymyxin B-induced skin hyperpigmentation is associated with its position $6 \mathrm{D}$-Phe, because no such reports have been published on colistin to date (35). It has been reported that penetration of polymyxins 
into CSF is a crucial indicator for optimizing in vivo efficacy and minimizing toxicity (28). However, the PK information of polymyxins in CSF after ITH/IVT delivery remains largely unknown. Our retrospective CSF determination showed a wide range of steady-state concentrations of polymyxin B (1.33-29.41 $\mathrm{mg} / \mathrm{L}$ ), which may be closely associated with rapid bacterial eradication and highly suspected adverse reactions.

The present case enriched the PK information of polymyxin B in CSF, which may improve the clinical practice of polymyxins, especially in pediatric patients. However, it has limitations; for example, only one case has been reported. Additionally, the therapeutic drug monitoring (TDM) of tigecycline and polymyxin B in CSF were retrospectively performed after the end of treatment without TDM-based dosing optimization. In the future, real-time $\mathrm{PK} / \mathrm{PD}$ data obtained from patients during ITH/IVT polymyxin B therapy should be required to optimize polymyxin use with maximal efficacy and minimal adverse effects.

\section{CONCLUSIONS}

This case highlights the issues involved in treating lifethreatening A. baumannii meningitis of pediatric patients. IV tigecycline should not be recommended for CNS infections of MDR Gram-negative pathogens because of the poor penetration into CSF. ITH administration of polymyxin B is a convenient route, especially in pediatric patients with lumbar drainage. Additionally, combined treatment with IV and ITH polymyxin $B$ is vital for CSF sterilization. However, the efficacy and safety of tigecycline and polymyxins for pediatric CNS infections need to be clarified. Given the narrow therapeutic windows of polymyxin $\mathrm{B}$, it is necessary to identify a practical $\mathrm{PK} / \mathrm{PD}$ profile for optimizing dosage regimens of ITH/IVT polymyxin therapy.

\section{DATA AVAILABILITY STATEMENT}

The original contributions presented in the study are included in the article/Supplementary Material, further inquiries can be directed to the corresponding author/s.

\section{ETHICS STATEMENT}

The studies involving human participants were reviewed and approved by the ethics committee of Daping Hospital.

\section{REFERENCES}

1. Tsuji BT, Pogue JM, Zavascki AP, Paul M, Daikos GL, Forrest A. International consensus guidelines for the optimal use of the polymyxins: endorsed by the American College of Clinical Pharmacy (ACCP), European Society of Clinical Microbiology and Infectious Diseases (ESCMID), Infectious Diseases Society of America (IDSA), International Society for Anti-infective Pharmacology (ISAP), Society of Critical Care Medicine (SCCM), and Society of Infectious Diseases Pharmacists (SIDP). Pharmacotherapy. (2019) 39:10-39. doi: 10.1002/ phar.2209
Written informed consent to participate in this study was provided by the participants' legal guardian/next of kin. Written informed consent was obtained from the individual(s), and minor(s)' legal guardian/next of kin, for the publication of any potentially identifiable images or data included in this article.

\section{AUTHOR CONTRIBUTIONS}

HX: conceptualization. CC: methodology. YZ: case presentation. YC: writing-original draft. XW: data analysis. DD: data collection. LX and MX: validation, writing-review, and editing. JC: supervision and project administration. All authors have read the last version of this manuscript and agree this submission.

\section{FUNDING}

This work was supported in part by grants from the Chongqing Federation of Social Sciences Circles (grant number 2018PY79) and the Training Program of Clinical Medical Researcher of Army Medical University (grant number 2018XLC3072). This project was also supported by the grants from the Innovation Ability Training Program of Army Specialized Medical Center (Grant No. 2019CXLCC013). The funders had no role in the design and conduct of this case, as well as no role in the preparation and approval of the manuscript.

\section{ACKNOWLEDGMENTS}

The authors would like to thank Dr. Ji Ming Wang of the Laboratory of Molecular Immunoregulation in the Cancer and Inflammation Program, Center for Cancer Research, National Cancer Institute at Frederick (Frederick, MD, USA) for reviewing and discussing of this manuscript. The authors would also like to thank all the members of the laboratory for the discussion and preparation of this manuscript.

\section{SUPPLEMENTARY MATERIAL}

The Supplementary Material for this article can be found online at: https://www.frontiersin.org/articles/10.3389/fped. 2021.564991/full\#supplementary-material

2. Tuon FF, Penteado-Filho SR, Amarante D, Andrade MA, Borba LA. Mortality rate in patients with nosocomial Acinetobacter meningitis from a Brazilian hospital. Braz J Infect Dis. (2010) 14:437-40. doi: 10.1016/S1413-8670(10)70090-8

3. Xiao JY, Zhang CM, Ye S. Acinetobacter baumannii meningitis in children: a case series and literature review. Infection. (2019) 47:643-49. doi: 10.1007/s15010-018-1234-1

4. Tsolaki V, Karvouniaris M, Manoulakas E, Kotlia P, Karadontas V, Fotakopoulos G, et al. Intraventricular CNS treatment with ColistinTigecycline combination: a case series. J Crit Care. (2018) 47:338-41. doi: $10.1016 /$ j.jcrc.2018.07.025 
5. Sharma R, Patel S, Abboud C, Diep J, Ly NS, Pogue JM, et al. Polymyxin $\mathrm{B}$ in combination with meropenem against carbapenemase-producing Klebsiella pneumoniae: pharmacodynamics and morphological changes. Int J Antimicrob Agents. (2017) 49:224-32. doi: 10.1016/j.ijantimicag.2016. 10.025

6. Guo W, Guo SC, Li M, Li LH, Qu Y. Successful treatment of extensively drug-resistant Acinetobacter baumannii ventriculitis with polymyxin B and tigecycline - a case report. Antimicrob Resist Infect Control. (2018) 7:22. doi: 10.1186/s13756-018-0313-5

7. Mizrahi CJ, Benenson S, Moscovici S, Candanedo C, Benifla M, Spektor S. Combination treatment with intravenous tigecycline and intraventricular and intravenous colistin in postoperative ventriculitis caused by multidrug-resistant Acinetobacter baumannii. Cureus. (2019) 11:e3888. doi: $10.7759 /$ cureus. 3888

8. Antachopoulos C, Karvanen M, Iosifidis E, Jansson B, Plachouras D, Cars O, et al. Serum and cerebrospinal fluid levels of colistin in pediatric patients. Antimicrob Agents Chemother. (2010) 54:3985-87. doi: 10.1128/AAC.01799-09

9. Karaiskos I, Galani L, Baziaka F, Giamarellou H. Intraventricular and intrathecal colistin as the last therapeutic resort for the treatment of multidrug-resistant and extensively drug-resistant Acinetobacter baumannii ventriculitis and meningitis: a literature review. Int J Antimicrob Agents. (2018) 41:499-508. doi: 10.1016/j.ijantimicag.2013.02.006

10. Clinical and Laboratory Standards Institute. CLSI. 2019. Performance Standards for Antimicrobial Susceptibility Testing, 29th Edn. CLSI Supplement M100. Clinical and Laboratory Standards Institute (CLSI) (2019).

11. US Food and Drug Administration. Antibacterial Susceptibility Test Interpretive Criteria. Available online at: https://www.fda.gov/Drugs/ DevelopmentApprovalProcess/DevelopmentResources/ucm575163.htm (accessed April 6, 2019).

12. Jenkins A, Thomson AH, Brown NM, Semple Y, Sluman C, Macgowan A, et al. Amikacin use and therapeutic drug monitoring in adults: do dose regimens and drug exposures affect either outcome or adverse events? A systematic review. J Antimicrob Chemother. (2016) 71:2754-9. doi: 10.1093/jac/dkw250

13. Kwon YS, Levin A, Kasperbauer SH, Huitt GA, Daley CL. Efficacy and safety of tigecycline for Mycobacterium abscessus disease. Respir Med. (2019) 158:89-91. doi: 10.1016/j.rmed.2019.10.006

14. Lauretti L, D’alessandris QG, Fantoni M, D'inzeo T, Fernandez E, Pallini $\mathrm{R}$, et al. First reported case of intraventricular tigecycline for meningitis from extremely drug-resistant Acinetobacter baumannii. J Neurosurg. (2017) 127:370-3. doi: 10.3171/2016.6.JNS16352

15. Zheng G, Cao L, Che Z, Mao E, Chen E, He J. Polymyxin B-induced skin hyperpigmentation: a rare case report and literature review. BMC Pharmacol Toxicol. (2018) 19:41. doi: 10.1186/s40360-018-0226-1

16. Mattos KPH, Gouvea IR, Quintanilha JCF, Cursino MA, Vasconcelos P, Moriel P. Polymyxin B clinical outcomes: a prospective study of patients undergoing intravenous treatment. J Clin Pharm Ther. (2019) 44:415-9. doi: $10.1111 /$ jcpt.12801

17. Tunkel AR, Hasbun R, Bhimraj A, Byers K, Kaplan SL, Scheld WM, et al. 2017 infectious diseases society of America’s Clinical Practice Guidelines for Healthcare-Associated Ventriculitis and Meningitis. Clin Infect Dis. (2017) 64:e34-e65. doi: 10.1093/cid/cix152

18. Chiotos K, Ross RK, Han JH, Miller M, Gerber JS. Use of carbapenems, polymyxins, and tigecycline in united states children's hospitals, 20102014. Open Forum Infect Dis. (2017) 4:ofx039. doi: 10.1093/ofid/ ofx039

19. Kanik-Yuksek S, Tezer H, Ozkaya-Parlakay A, Gulhan B, Sayed-Oskovi H, Kara A, et al. Multidrug-resistant Acinetobacter baumannii bacteremia treated with tigecycline in two pediatric burn patients. Pediatr Infect Dis J. (2015) 34:677. doi: 10.1097/INF.0000000000000699

20. Emiroglu M, Alkan G, Turk Dagi H. Tigecycline therapy in an infant for ventriculoperitoneal shunt meningitis. Pediatrics. (2017) 139:e20160963. doi: 10.1542/peds.2016-0963

21. Tutuncu EE, Kuscu F, Gurbuz Y, Ozturk B, Haykir A, Sencan I. Tigecycline use in two cases with multidrug-resistant Acinetobacter baumannii meningitis. Int J Infect Dis. (2010) 14(Suppl 3):e224-6. doi: 10.1016/j.ijid.2009.07.022

22. Kooli I, Brahim HB, Kilani M, Gannouni C, Aouam A, Toumi A, et al. Successful treatment of postoperative multidrug-resistant Acinetobacter baumannii meningitis by tigecycline. J Glob Antimicrob Resist. (2016) 5:62-3. doi: 10.1016/j.jgar.2015.12.003

23. Purdy J, Jouve S, Yan JL, Balter I, Dartois N, Cooper CA, et al Pharmacokinetics and safety profile of tigecycline in children aged 8 to 11 years with selected serious infections: a multicenter, openlabel, ascending-dose study. Clin Ther. (2012) 34:496-507.e491. doi: 10.1016/j.clinthera.2011.12.010

24. Karageorgopoulos DE, Kelesidis T, Kelesidis I, Falagas ME. Tigecycline for the treatment of multidrug-resistant (including carbapenem-resistant) Acinetobacter infections: a review of the scientific evidence. J Antimicrob Chemother. (2008) 62:45-55. doi: 10.1093/jac/dkn165

25. Wu Y, Chen K, Zhao J, Wang Q, Zhou J. Intraventricular administration of tigecycline for the treatment of multidrug-resistant bacterial meningitis after craniotomy: a case report. J Chemother. (2018) 30:49-52. doi: 10.1080/1120009X.2017.1338846

26. Ye S, Zhang C, Lin S. Preliminary experience with tigecycline treatment for severe infection in children. Eur J Pediatr. (2018) 177:1489-96. doi: 10.1007/s00431-018-3208-9

27. Nation RL, Forrest A. Clinical pharmacokinetics, pharmacodynamics and toxicodynamics of polymyxins: implications for therapeutic use. Adv Exp Med Biol. (2019) 1145:219-49. doi: 10.1007/978-3-030-16373-0_15

28. Velkov T, Dai C, Ciccotosto GD, Cappai R, Hoyer D, Li J. Polymyxins for CNS infections: pharmacology and neurotoxicity. Pharmacol Ther. (2018) 181:85-90. doi: 10.1016/j.pharmthera.2017. 07.012

29. Khan SA, Waqas M, Siddiqui UT, Shamim MS, Nathani KR, Jooma $\mathrm{R}$, et al. Intrathecal and intraventricular antibiotics for postoperative Gram-negative meningitis and ventriculitis. Surg Neurol Int. (2017) 8:226. doi: 10.4103/sni.sni_81_17

30. Ozdemir H, Tapisiz A, Ciftci E, Ince E, Mokhtari H, Guriz H, et al. Successful treatment of three children with post-neurosurgical multidrugresistant Acinetobacter baumannii meningitis. Infection. (2010) 38:241-4. doi: 10.1007/s15010-010-0018-z

31. Pogue JM, Lee J, Marchaim D, Yee V, Zhao JJ, Chopra T, et al. Incidence of and risk factors for colistin-associated nephrotoxicity in a large academic health system. Clin Infect Dis. (2011) 53:879-84. doi: 10.1093/cid/cir611

32. Falagas ME, Bliziotis IA, Tam VH. Intraventricular or intrathecal use of polymyxins in patients with Gram-negative meningitis: a systematic review of the available evidence. Int J Antimicrob Agents. (2007) 29:9-25. doi: 10.1016/j.ijantimicag.2006.08.024

33. Falagas ME, Kasiakou SK. Toxicity of polymyxins: a systematic review of the evidence from old and recent studies. Crit Care. (2006) 10:R27. doi: $10.1186 /$ cc3995

34. Lahiry S, Choudhury S, Mukherjee A, Bhunya PK, Bala M. Polymyxin B-induced diffuse cutaneous hyperpigmentation. J Clin Diagn Res. (2017) 11:FD01-F2. doi: 10.7860/JCDR/2017/24278.9213

35. Nang SC, Azad, MAK, Velkov T, Zhou QT, Li J. Rescuing the last-line polymyxins: achievements and challenges. Pharmacol Rev. (2021) 73:679-728. doi: 10.1124/pharmrev. 120.000020

Conflict of Interest: The authors declare that the research was conducted in the absence of any commercial or financial relationships that could be construed as a potential conflict of interest.

Publisher's Note: All claims expressed in this article are solely those of the authors and do not necessarily represent those of their affiliated organizations, or those of the publisher, the editors and the reviewers. Any product that may be evaluated in this article, or claim that may be made by its manufacturer, is not guaranteed or endorsed by the publisher.

Copyright $\odot 2021$ Xing, Cheng, Zhang, Cai, Wang, Deng, Xu, Xu and Chen. This is an open-access article distributed under the terms of the Creative Commons Attribution License (CC BY). The use, distribution or reproduction in other forums is permitted, provided the original author(s) and the copyright owner(s) are credited and that the original publication in this journal is cited, in accordance with accepted academic practice. No use, distribution or reproduction is permitted which does not comply with these terms. 